\title{
Studying the nature of the unidentified gamma-ray source HESS J1841-055 with the MAGIC telescopes
}

\author{
MAGIC Collaboration: V. A. Acciari, ${ }^{1}$ S. Ansoldi, ${ }^{2}$ L. A. Antonelli, ${ }^{3}$ A. Arbet Engels, ${ }^{4}$ K. Asano, ${ }^{5}$ \\ D. Baack, ${ }^{6}$ A. Babić, ${ }^{7}$ B. Banerjee,${ }^{8}$ A. Baquero, ${ }^{9}$ U. Barres de Almeida, ${ }^{10}$ J. A. Barrio, ${ }^{9}$ J. Becerra
} González, ${ }^{1,11}$ W. Bednarek, ${ }^{12}$ L. Bellizzi, ${ }^{13}$ E. Bernardini, ${ }^{14}$ M. Bernardos, ${ }^{15}$ A. Berti, ${ }^{16}$ J. Besenrieder,${ }^{17}$ W. Bhattacharyya, ${ }^{14}$ C. Bigongiari, ${ }^{3}$ A. Biland, ${ }^{4}$ O. Blanch, ${ }^{18}$ G. Bonnoli, ${ }^{13}$ Ž. Bošnjak, ${ }^{7}$ G. Busetto, ${ }^{19}$ R. Carosi, ${ }^{20}$ G. Ceribella, ${ }^{17}$ M. Cerruti, ${ }^{21}$ Y. Chai, ${ }^{17}$ A. Chilingarian, ${ }^{22}$ S. Cikota, ${ }^{7}$ S. M. Colak, ${ }^{18}$ E. Colombo, ${ }^{1}$ J. L. Contreras, ${ }^{9}$ J. Cortina, ${ }^{15}$ S. Covino, ${ }^{3}$ G. D’ Amico, ${ }^{17}$ V. D'Elia, ${ }^{3}$ P. Da Vela,${ }^{20}$ F. Dazzi, ${ }^{3}$ A. De Angelis,${ }^{19}$ B. De Lotto,${ }^{2}$ M. Delfino,${ }^{18} \dagger$ J. Delgado,,${ }^{18} \dagger$ C. Delgado Mendez, ${ }^{15}$ D. Depaoli, ${ }^{16}$ T. Di Girolamo, ${ }^{16}$ F. Di Pierro, ${ }^{16}$ L. Di Venere, ${ }^{16}$ E. Do Souto Espiñeira, ${ }^{18}$ D. Dominis Prester, ${ }^{23}$ A. Donini, ${ }^{2}$ D. Dorner, ${ }^{24}$ M. Doro, ${ }^{19}$ D. Elsaesser, ${ }^{6}$ V. Fallah Ramazani, ${ }^{25}$ A. Fattorini, ${ }^{6}$ G. Ferrara, ${ }^{3}$ L. Foffano, ${ }^{19}$ M. V. Fonseca, ${ }^{9}$ L. Font, ${ }^{26}$ C. Fruck, ${ }^{17}$ S. Fukami, ${ }^{5}$ R. J. García López, ${ }^{1,11}$ M. Garczarczyk, ${ }^{14}$ S. Gasparyan, ${ }^{22}$ M. Gaug, ${ }^{26}$ N. Giglietto, ${ }^{16}$ F. Giordano, ${ }^{16}$ P. Gliwny, ${ }^{12}$ N. Godinović, ${ }^{27}$ D. Green, ${ }^{17}$ D. Hadasch, ${ }^{5}$ A. Hahn, ${ }^{17}$ L. Heckmann, ${ }^{17}$ J. Herrera, ${ }^{1,11}$ J. Hoang, ${ }^{9}$ D. Hrupec,${ }^{28}$ M. Hütten, ${ }^{17}$ T. Inada, ${ }^{5}$ S. Inoue,${ }^{5}$ K. Ishio, ${ }^{17}$ Y. Iwamura, ${ }^{5}$ L. Jouvin, ${ }^{18}$ Y. Kajiwara, ${ }^{29}$ M. Karjalainen, ${ }^{1}$ D. Kerszberg, ${ }^{18}$ Y. Kobayashi, ${ }^{5}$ H. Kubo, ${ }^{29}$ J. Kushida, ${ }^{30}$ A. Lamastra, ${ }^{3}$ D. Lelas, ${ }^{27}$ F. Leone, ${ }^{3}$ E. Lindfors,${ }^{25}$ S. Lombardi,${ }^{3}$ F. Longo,${ }^{2} \ddagger$ M. López, ${ }^{9}$ R. López-Coto, ${ }^{19}$ A. López-Oramas, ${ }^{1,11 \star ~ S . ~ L o p o r c h i o, ~}{ }^{16}$ B. Machado de Oliveira Fraga, ${ }^{10}$ C. Maggio, ${ }^{26}$ P. Majumdar, ${ }^{8}$ M. Makariev, ${ }^{31}$ M. Mallamaci, ${ }^{19}$ G. Maneva, ${ }^{31}$ M. Manganaro, ${ }^{23}$ K. Mannheim, ${ }^{24}$ L. Maraschi, ${ }^{3}$ M. Mariotti, ${ }^{19}$ M. Martínez, ${ }^{18}$ D. Mazin, ${ }^{5}$ S. Mender, ${ }^{6}$ S. Mićanović, ${ }^{23}$ D. Miceli, ${ }^{2}$ T. Miener, ${ }^{9}$ M. Minev, ${ }^{31}$ J. M. Miranda, ${ }^{13}$ R. Mirzoyan, ${ }^{17}$ E. Molina, ${ }^{21}$ A. Moralejo, ${ }^{18}$ D. Morcuende, ${ }^{9}$ V. Moreno, ${ }^{26}$ E. Moretti, ${ }^{18}$ P. Munar-Adrover ${ }^{26}$ V. Neustroev, ${ }^{32}$ C. Nigro, ${ }^{18} \mathrm{~K}$. Nilsson, ${ }^{25}$ D. Ninci, ${ }^{18}$ K. Nishijima, ${ }^{30}$ K. Noda, ${ }^{5}$ S. Nozaki, ${ }^{29}$ Y. Ohtani, ${ }^{5}$ T. Oka, ${ }^{29}$ J. Otero-Santos, ${ }^{1,11}$ M. Palatiello, ${ }^{2}$ D. Paneque, ${ }^{17}$ R. Paoletti, ${ }^{13}$ J. M. Paredes, ${ }^{21}$ L. Pavletić, ${ }^{23}$ P. Peñil, ${ }^{9}$ C. Perennes, ${ }^{19}$ M. Persic, ${ }^{2} \S$ P. G. Prada Moroni, ${ }^{20}$ E. Prandini, ${ }^{19}$ C. Priyadarshi, ${ }^{18}$ I. Puljak, ${ }^{27}$ W. Rhode,${ }^{6}$ M. Ribó, ${ }^{21}$ J. Rico, ${ }^{18}$ C. Righi, ${ }^{3}$ A. Rugliancich, ${ }^{20}$ L. Saha, ${ }^{\star \star}$ N. Sahakyan, ${ }^{22}$ T. Saito, ${ }^{5}$ S. Sakurai, ${ }^{5}$ K. Satalecka, ${ }^{14}$ B. Schleicher, ${ }^{24}$ K. Schmidt, ${ }^{6}$ T. Schweizer, ${ }^{17}$ J. Sitarek, ${ }^{12}$ I. Šnidarić, ${ }^{33}$ D. Sobczynska, ${ }^{12}$ A. Spolon, ${ }^{19}$ A. Stamerra, ${ }^{3}$ D. Strom, ${ }^{17}$ M. Strzys,${ }^{5}$ Y. Suda, ${ }^{17}$ T. Surić, ${ }^{33}$ M. Takahashi, ${ }^{5}$ F. Tavecchio, ${ }^{3}$ P. Temnikov, ${ }^{31}$ T. Terzić, ${ }^{23}$ M. Teshima, ${ }^{17}$ N. Torres-Albà, ${ }^{21}$ L. Tosti, ${ }^{16}$ S. Truzzi, ${ }^{13}$ J. van Scherpenberg, ${ }^{17}$ G. Vanzo,, 11 M. Vazquez Acosta, ${ }^{1,11}$ S. Ventura, ${ }^{13}$ V. Verguilov, ${ }^{31}$ C. F. Vigorito, ${ }^{16}$ V. Vitale, ${ }^{16}$ I. Vovk, ${ }^{5}$ M. Will ${ }^{17}$ and D. Zarić ${ }^{27}$

Affiliations are listed at the end of the paper 


\section{ABSTRACT}

We investigate the physical nature and origin of the gamma-ray emission from the extended source HESS J1841-055 observed at $\mathrm{TeV}$ and $\mathrm{GeV}$ energies. We observed HESS J1841-055 at TeV energies for a total effective time of $43 \mathrm{~h}$ with the MAGIC telescopes, in 2012 and 2013. Additionally, we analysed the GeV counterpart making use of about 10 yr of Fermi-LAT data. Using both Fermi-LAT and MAGIC, we study both the spectral and energy-dependent morphology of the source for almost four decades of energy. The origin of the gamma-ray emission from this region is investigated using multiwaveband information on sources present in this region, suggested to be associated with this unidentified gamma-ray source. We find that the extended emission at GeV-TeV energies is best described by more than one source model. We also perform the first energy-dependent analysis of the HESS J1841-055 region at GeV-TeV. We find that the emission at lower energies comes from a diffuse or extended component, while the major contribution of gamma rays above $1 \mathrm{TeV}$ arises from the southern part of the source. Moreover, we find that a significant curvature is present in the combined observed spectrum of MAGIC and Fermi-LAT. The first multiwavelength spectral energy distribution of this unidentified source shows that the emission at GeV-TeV energies can be well explained with both leptonic and hadronic models. For the leptonic scenario, bremsstrahlung is the dominant emission compared to inverse Compton. On the other hand, for the hadronic model, gamma-ray resulting from the decay of neutral pions $\left(\pi^{0}\right)$ can explain the observed spectrum. The presence of dense molecular clouds overlapping with HESS J1841-055 makes both bremsstrahlung and $\pi^{0}$-decay processes the dominant emission mechanisms for the source.

Key words: ISM: individual objects: HESS J1841-055 - ISM: supernova remnants-gamma-rays: stars-radiation mechanisms: non-thermal - pulsars: general.

\section{INTRODUCTION}

The unidentified gamma-ray source HESS J1841-055 was first discovered at TeV energies in 2007 by the High Energy Stereoscopic System (HESS) during the Galactic plane survey (Aharonian et al. 2008). The observed emission was reported as extended with an elliptical extension of 0.41 and 0.25 along the semimajor and semiminor axes, respectively, and centred at Right Ascension (RA) $18^{\mathrm{h}} 40^{\mathrm{m}} 55^{\mathrm{s}}$ and declination (Dec.) $5^{\circ} 33^{\prime} 00^{\prime \prime}$ with a position angle $39^{\circ}$ relative to the RA axis. HESS J1841-055 was detected with a statistical significance of $10.7 \sigma$ and a flux of $(12.8 \pm 1.3) \times 10^{-12}$ $\mathrm{cm}^{-2} \mathrm{~s}^{-1}$ between 0.54 and $80 \mathrm{TeV}$. The spectrum is best described by a power law (PL) with a spectral index of $2.41 \pm 0.1_{\text {stat }} \pm 0.2_{\text {sys }}$. These results are compatible with the recent results reported by HESS collaboration (HESS Collaboration et al. 2018). Using the ARGOYBJ experiment for energies above $0.9 \mathrm{TeV}$, Bartoli et al. (2013) reported a similar extension as seen be the HESS collaboration but a three times larger flux due to differing background estimation techniques between the experiments. This region was further investigated by the HAWC observatory also at $\mathrm{TeV}$ energies. The source $1 \mathrm{HWC}$ J1838-060, from the First HAWC Catalog, was detected at $6.1 \sigma$ post-trial significance. It is located in the middle of HESS J1841-055 and another known TeV source, HESS J1837-069 (Abeysekara et al. 2016). This detection by HAWC was found to be overlapping with the extension of HESS J1841-055, and the differential flux normalization was compatible with the one reported by the HESS collaboration. The second HAWC Catalog also revealed a source, 2 HWC J1837-065, which was likely to be associated with HESS J1841-055 (Abeysekara et al. 2017). Its spectral index varies from $-2.90 \pm 0.04$ for a point-like emission to $-2.66 \pm 0.03$ for a $2^{\circ}$ radius.

This region was further investigated at other wavelengths to search for possible counterparts. Although no confirmed counterparts of the TeV source HESS J1841-055 at lower energies are known, several possible associations have been suggested. The emission from HESS J1841-055 may be due to either a single extended source or several unresolved sources. Sguera et al. (2009), making use of INTEGRAL data, proposed as counterpart the unidentified transient source 3EG J1837-0423, which was likely to be associated to the Supergiant Fast X-ray Transient (SFXT) AX J1841.0-0536. At X-ray energies, observations of this extended region were done with SUZAKU and an $\mathrm{X}$-ray source was discovered (Nobukawa et al. 2015). The detection of two separate extended sources (FGES J1839.4-0554 and FGES J1841.4-0514) was also reported in this region at energies above $10 \mathrm{GeV}$ using data from the Fermi-Large Area Telescope (LAT; Ackermann et al. 2017; Ajello et al. 2017). Some potential sources at different wavelengths suggested to be associated with HESS $\mathrm{J} 1841-055$ is discussed later in detail.

In this paper, we study this complex region using dedicated observations with the MAGIC telescopes at $\mathrm{TeV}$ energies. We also explore the $\mathrm{GeV}$ counterpart making use of 10-yr data of Fermi-LAT. We finally model the GeV-TeV emission to unveil the dominant gammaray emission mechanisms at work. The potential counterparts at other frequencies are also investigated. The low-energy (LE) threshold of MAGIC, which allows to overlap with Fermi-LAT in the GeV domain, combined with the MAGIC capabilities of reaching several $\mathrm{TeV}$, make the MAGIC telescopes a suitable instrument to study this region within a broad energy range. The combination of both MAGIC and Fermi-LAT allows spectral studies of this complex region for almost four decades in energy.

This paper is organized as follows: In Section 2, we describe the detailed analyses of the MAGIC and Fermi-LAT data. The results are discussed in Section 3. Potential counterparts are proposed in Section 4. The multiwaveband modelling of the source is explained in Section 5. Finally, we summarize and conclude in Section 6.

\section{OBSERVATIONS AND DATA REDUCTION}

\subsection{MAGIC}

Very High Energy (VHE, $E>100 \mathrm{GeV}$ ) gamma-ray observations of HESS J1841-055 are performed using the MAGIC telescopes. MAGIC consists of two 17-m diameter Imaging Atmospheric Cherenkov Telescopes (IACTs) located at the Observatory of Roque de los Muchachos (28.8 N, 17.9 W, $2200 \mathrm{~m}$ above the sea level) on the Canary Island of La Palma, Spain. The energy threshold of the MAGIC stereoscopic system is about $50 \mathrm{GeV}$, and it is able to detect $\sim 0.6$ per cent of the Crab Nebula flux above $250 \mathrm{GeV}$ at $5 \sigma$ significance in $50 \mathrm{~h}$ of observations at small $\left(<30^{\circ}\right)$ zenith angles (Aleksić et al. 2012). HESS J1841-055 was observed between 2012 
April 2012 and 2013 August, for a total of about 43 h, at zenith angles between $5^{\circ}$ and $50^{\circ}$, resulting in an energy threshold for this analysis of $\sim 150 \mathrm{GeV}$. To estimate the background simultaneously with the source data, the observations are performed in the so-called wobble mode (Fomin et al. 1994) at two symmetrical positions, with the source located 0.55 off-axis from the centre of the camera. After quality cuts, which account for hardware problems, unusual rates, and bad atmospheric conditions, $\sim 34 \mathrm{~h}$ of high-quality, dark-time data are selected for further analysis. The analysis of the MAGIC data is performed using the standard MAGIC Analysis and Reconstruction Software (MARS; Moralejo et al. 2009; Zanin et al. 2013) and standard analysis procedure.

Given the extension of the source and the possibility of contamination from other nearby sources, we study the region using an iterative maximum likelihood method included in the SKYPRISM package (Vovk, Strzys \& Fruck 2018). SKYPRISM has specifically been developed to perform 2D fitting of IACTs data and has been optimized for MAGIC data. This set of tools compute the instrument response function (IRF) and perform a simultaneous maximum likelihood fit of source models of arbitrary morphology to the sky images. With SKYPRISM, it is then possible to analyse MAGIC data of extended sources of arbitrary morphology and multiple, overlapping sources.

We compute the event count map, the background map, and the instrument response functions that include point spread function (PSF), energy migration matrix, and exposure map. We use the 'exclusion map' method for generating the background map excluding a circular region of 0.5 around the centre of the HESS $\mathrm{J} 1841-055(\mathrm{RA}=280.23$, Dec. $=-5.55)$ and a circular region of 0.3 around a bright spot at the southern edge of the camera (RA = 279.4 , Dec. $=-6.45$ ). A user-defined source model (2D Gaussian) is used to fit the measured event maps for maximizing the loglikelihood estimate. To calculate the individual spectral parameters of the sources obtained from the modelling of the region, we use the maximum log-likelihood method, as defined in Vovk et al. (2018), assuming a PL model for the source at the pivot energy $1 \mathrm{TeV}$ (energy at which the uncertainty in the normalization is minimum). The observations for this work are performed at low and medium zenith angles $\left(Z<50^{\circ}\right)$. Given the very high signal-to-noise ratio $(>0.4)$, the systematic uncertainties can be considered similar to those reported in Aleksić et al. (2012), defined as 12 percent in the integral flux for stereoscopic observations.

\subsection{Fermi-LAT}

The LAT, onboard the Fermi Gamma Ray Space Telescope, allows for the detection of gamma rays from $30 \mathrm{MeV}$ to $>500 \mathrm{GeV}$ with its large effective area and wide field of view (Atwood et al. 2009). In our analysis, we select nearly 10 yr (i.e. from 2008 September 1 to 2017 May 5) of Pass 8 SOURCE class (P8R3) LAT events in the reconstructed energy of about $10 \mathrm{GeV}$ to $1 \mathrm{TeV}$ within a $15^{\circ}$ region of interest (ROI) around the fourth Fermi-LAT catalogue source 4FGL J1840.9-0532e (associated to 3FHL J1840.9-0532e). TeV source HESS J1841-055 is associated with the Fermi-LAT source 4FGL J1840.9-0532e. The Fermi Science Tools (FST) analysis package ${ }^{1}$ version V11r5p3 and the P8R3_SOURCE_V2 IRFs are used for the analysis. We also use the PYTHON-based package fermipy (version $0.17 .4^{2}$ ) to facilitate the analysis of data with the FST.

${ }^{1}$ https://fermi.gsfc.nasa.gov/ssc/data/analysis/software/.

${ }^{2}$ https://fermipy.readthedocs.io/en/latest/.
We select photons of energies greater than $10 \mathrm{GeV}$ with arrival direction within $105^{\circ}$ from local zenith to remove contamination from the Earth's emission. The PASS 8 source class allows for the use of four different event types that are based on the event-byevent quality of reconstructed direction (PSF) and energy. Hence, the data are separated into these event types to optimize selection of events based on the quality of reconstruction of direction of incoming photons and energy. The Galactic diffuse emission is modeled with the standard Fermi-LAT diffuse emission model ( $g l l_{-}$iem_v07.fits). The isotropic emission from extragalactic radiation and residual background models (iso_P8R3_SOURCE_V2_PSF[0/1/2/3]_v1.txt) are also used corresponding to four event types.

We first start with a baseline sky model that includes all 4FGL point and extended sources within the ROI listed in the 4FGL catalogue ${ }^{3}$ (Abdollahi et al. 2020). The extended source 4FGL J1840.9-0532e (associated with 3FHL J1840.9-0532e) is our source of interest in the ROI that is associated with two sources from the Fermi Galactic Extended Source Catalog (FGES; Ackermann et al. 2017) and it is included in the model. Initially, we use the baseline model to optimize parameters of the sources by fitting their flux and spectral parameters. After the initial optimization, we remove all sources for which the values of the predicted number of counts in the model, Npred, are less than 2.0 and we free spectral shapes and normalizations for all the sources that lie within $3^{\circ}$ from the centre of the ROI. The isotropic diffused background model is fixed to the value obtained after the first optimization of the ROI but the diffuse Galactic model is kept free for all different configurations or models discussed below. Then we use the binned maximum likelihood method to estimate the best-fitting model parameters using a $15 \times 15 \mathrm{deg}^{2}$ region centred on 4FGL J1840.9-0532e with a spatial bin-size of 0.06 and 10 equally spaced energy bins per decade of energy. We then relocate the source of interest using the maximum likelihood method to find the best source position. As the next step, we use an iterative maximum likelihoodbased source finding algorithm to identify new point sources within 0.5 from the centre of the ROI. The algorithm finds point sources within this ROI with test statistics, ${ }^{4} \mathrm{TS}>16$. We continue searching for new sources until all the point sources are added to the baseline model. Following this, we remove all the sources with $\mathrm{TS}<16$ from the ROI and perform the maximum likelihood method for the best-fitting model parameters.

\section{RESULTS}

\subsection{MAGIC}

\subsubsection{Morphology}

In order to study the energy-dependent morphology of the extended source HESS J1841-055, we produce skymaps for different energy ranges using SKYPRISM. Fig. 1 shows the relative flux skymaps, with $3 \sigma$ and $5 \sigma$ contours extracted from the TS map, produced for energies 50-500 GeV (LE, map), $500 \mathrm{GeV}-1 \mathrm{TeV}$ (medium energy, ME, map), and $>1 \mathrm{TeV}$ (high energy, HE, map), respectively. The relative flux is defined as the excess events divided by the background events. To calculate the extension of the source in each of the energy ranges,

\footnotetext{
${ }^{3}$ https://fermi.gsfc.nasa.gov/ssc/data/access/lat/8yr_catalog/gll_psc_v19.fit. ${ }^{4}$ The test statistic (TS) of a source is evaluated using a likelihood ratio test defined as TS $=-2 \log \left(\mathcal{L}_{1} / \mathcal{L}_{0}\right)$, where $\mathcal{L}_{0}$ and $\mathcal{L}_{1}$ are the likelihoods of the background model without the source (null hypothesis) and the hypothesis being tested (source plus background), respectively. The detection significance is approximately the square root of the TS.
} 

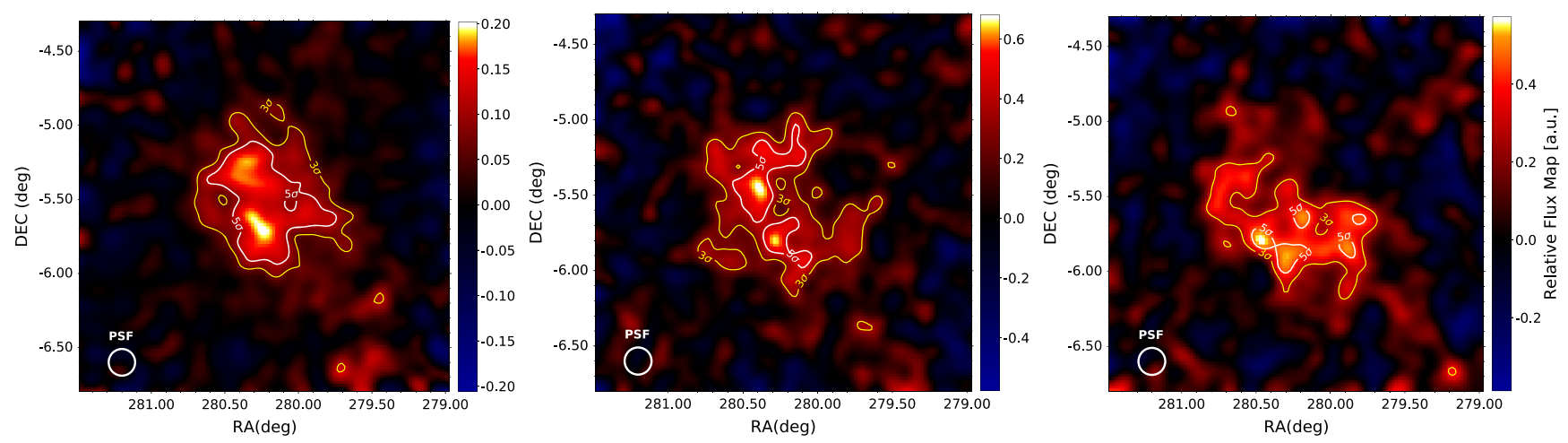

Figure 1. Energy-dependent gamma-ray relative flux maps with $3 \sigma$ (yellow) and $5 \sigma$ (white) contour levels of the extended source HESS J1841-055 as seen by MAGIC. The energy ranges covered are LE ( $50-500 \mathrm{GeV}), \mathrm{ME}(500 \mathrm{GeV}-1 \mathrm{TeV})$, and $\mathrm{HE}(>1 \mathrm{TeV})$, shown in the three panels, from the left- to right-hand side, respectively.

Table 1. Best-fitting parameters of the extension of the source measured by MAGIC considering a symmetrical 2D Gaussian model.

\begin{tabular}{lccc}
\hline Energy range & $\mathrm{RA}\left({ }^{\circ}\right)$ & Dec. $\left({ }^{\circ}\right)$ & Extension $\left({ }^{\circ}\right)$ \\
\hline $50-500 \mathrm{GeV}$ & $280.27_{-0.04}^{+0.03}$ & $-5.59_{-0.03}^{+0.02}$ & $0.39_{-0.15}^{+0.21}$ \\
$500-1000 \mathrm{GeV}$ & $280.29_{-0.04}^{+0.01}$ & $-5.58_{-0.05}^{+0.01}$ & $0.42_{-0.19}^{+0.04}$ \\
$>1000 \mathrm{GeV}$ & $280.29_{-0.04}^{+0.01}$ & $-5.70_{-0.05}^{+0.01}$ & $0.45_{-0.04}^{+0.04}$ \\
\hline
\end{tabular}

we consider a radially symmetrical two-dimensional (2D) Gaussian shape. The $1 \sigma$ standard deviation of the Gaussian is considered to be the extension or radius of the source. The radius is kept as a free parameter and is allowed to change by 0.01 over a range of $0.1-0.6$ during the maximum likelihood fitting. Moreover, we simultaneously keep changing the origin of the Gaussian by changing RA and Dec. by 0.01 for both of them over a range of $1^{\circ}$. The best-fitting locations along with the extensions of the source for different energy ranges are shown in Table 1. The extension of the source at these three energy ranges appears to be the same; however, the overall detection significance of the extended emission reduces at higher energies, revealing only a few hotspots in the southern part of the source (see Fig. 1). The fitted extension is the same (within errors) in the whole energy range (see Table 1). MAGIC observations show that the source has an extension compatible with that measured by HESS collaboration at $\mathrm{TeV}$ energies. It is also evident from the different maps that the extended region shows several bright hotspots with a significance of more than $5 \sigma$. Many bright highly significant spots are detected at LE and ME energies, while they mostly disappear at HE. These hotspots hint the presence of multiple sources in the region. It also indicates that the most significant emission at higher energies is coming from the southern part of the region.

As discussed above, the extended source HESS J1841-055 may potentially consist of multiple sources. To check this, we consider three different source models covering the full energy range, i.e. energies from $50 \mathrm{GeV}$ to above $1 \mathrm{TeV}$. We first consider a singlesource model where the extended source is considered to be a $2 \mathrm{D}$ elliptical Gaussian. We leave the peak position, extension along $x$ - and $y$-direction and angle with respect to the $x$-direction free while maximizing the likelihood value of the fit. For the second model, we replace the single-source model with two sources that are modelled as 2D circular Gaussian. The peak position and radius ( $1 \sigma$ standard deviation) of the two sources are free parameters of
Table 2. Significance of the multiple sources at $\mathrm{TeV}$ energies for different spatial source models.

\begin{tabular}{lcc}
\hline Spatial model & $\Delta \log \mathcal{L}^{a}$ & d.o.f \\
\hline One elliptical Gaussian model & 0.0 & 5 \\
Two Gaussian models & 4.7 & 6 \\
Two Gaussian + one elliptical disc models & 8.1 & 11 \\
\hline
\end{tabular}

Note. ${ }^{a}$ Calculated with respect to the one-source model.

the model. Finally, for the third option, we model the entire source region considering three different sources, one with elliptical disc model and the other two with Gaussian models. The results of the maximum likelihood values are given in Table 2. It is found that both two-source model and three-source models are better than a single-source model. The improvement of the two-source model with respect to to the one-source model is given by TS $=9.4$ for 1 additional degree of freedom (d.o.f.), which corresponds to an improvement at $3 \sigma$. The improvement of the three-source model with respect to to the one-source model is given by test statistics of 16.2 for additional 5 d.o.f., which corresponds to an improvement of $2.7 \sigma$. This hints that the HESS J1841-055 region is better modelled by multiple sources. The parameters of the best-fitting models are shown in Table 3.

\subsubsection{Spectrum}

The spectral energy distribution (SED) is calculated in the energy range of $50 \mathrm{GeV}$ to $>1 \mathrm{TeV}$, using the SKYPRISM package. We consider the extended 2D Gaussian template with the extension 0.4 at the position of the HESS J1841-055 and an isotropic background. The assumed spectrum of the source is considered to follow a simple PL model, which is defined as follows:

$\mathrm{PL}: \frac{\mathrm{d} N}{\mathrm{~d} E}=N_{0}\left(\frac{E}{E_{0}}\right)^{-\alpha}$,

where $N_{o}$ and $\alpha$ are parameters of the model. The best-fitting spectral parameters are $N_{\mathrm{o}}=(9.43 \pm 0.29) \times 10^{-12} \mathrm{TeV}^{-1} \mathrm{~cm}^{-2} \mathrm{~s}^{-1}, \alpha=$ $2.57 \pm 0.05$. The gamma-ray flux above $50 \mathrm{GeV}$ is $F(>50 \mathrm{GeV})=$ $2.23 \times 10^{-10} \mathrm{~cm}^{-2} \mathrm{~s}^{-1}$. The SED measured by MAGIC is plotted in Fig. 2.

Although morphology studies reveal that the emission region can be modelled better with more than one source, we cannot make any 
Table 3. Parameters of the best-fitting single and multisource models.

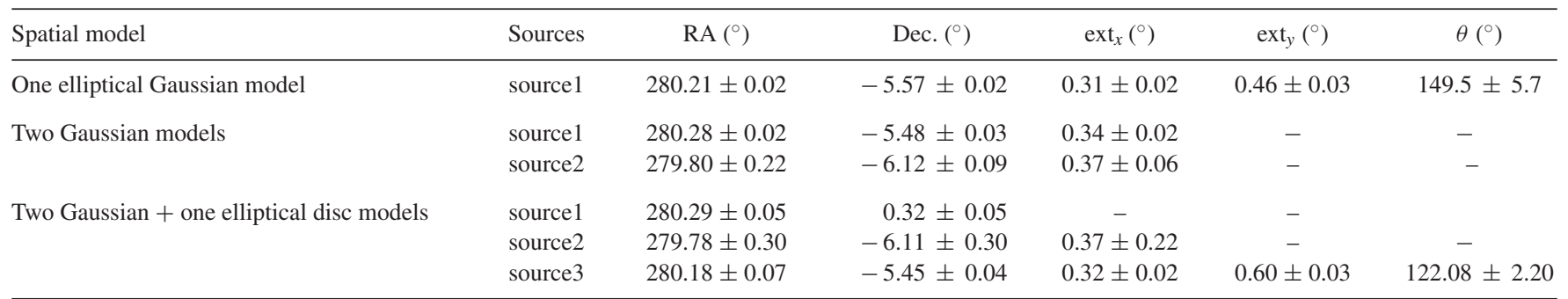

Notes. The ext $x$ and ext $y$ are extensions of the models along $x$ - and $y$-directions, respectively. For the elliptical Gaussian model, they are standard deviations, whereas for the elliptical disc model, they correspond to semimajor and semiminor axes, respectively.

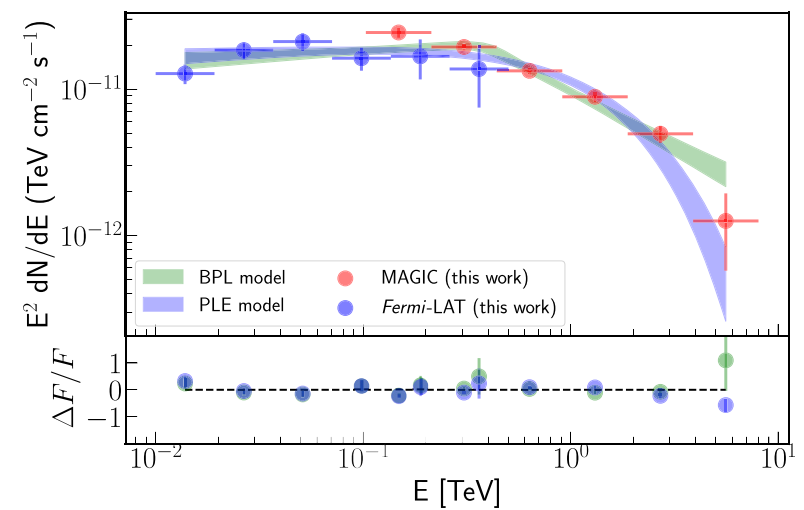

Figure 2. The SED of the extended source HESS J1841-055. The MAGIC energy fluxes are shown for energy above $100 \mathrm{GeV}$ (red) whereas Fermi-LAT energy fluxes are obtained for energy above $10 \mathrm{GeV}$ (blue). The combined fit of the Fermi-LAT and MAGIC SEDs is best described by either a BPL model (green shaded region) or a PLE (blue shaded region) model.

robust estimate on the number of distinct sources due to limitations of the software tool. Hence, we do not provide high-quality SEDs associated with these sources.

\subsection{Fermi-LAT}

\subsubsection{Morphology}

For the morphological analysis of the source, photons with energy above $10 \mathrm{GeV}$ up to $1 \mathrm{TeV}$ are considered to reduce the contamination from nearby pulsars within the ROI. With the baseline model, as discussed in Section 2.2, we perform the binned maximum likelihood analysis on 4FGL J1840.9-0532e and find the best-fitting model parameters. To estimate the size of 4FGL J1840.9-0532e, we calculate the TS of the extension $\left(\mathrm{TS}_{\mathrm{ext}}\right)$ parameter, which is the likelihood ratio of the likelihood for being a point-like source $\left(L_{\mathrm{pt}}\right)$ to a likelihood for an assumed extension $\left(L_{\mathrm{ext}}\right), \mathrm{TS}_{\mathrm{ext}}=2 \log \left(L_{\mathrm{ext}} / L_{\mathrm{pt}}\right)$. In order to test the extension of the source of interest, a radially symmetric Gaussian is considered and we vary its $\sigma$ from 0.01 to 1.5 in steps of 0.1 . We also simultaneously leave the location of the centre of the source free within $1 \sigma$ extension of the Gaussian. We find that the source extension is $0.64 \pm 0.11$ with the $T_{\mathrm{ext}}=264$, which corresponds to a significance of about $16 \sigma$. We also consider a radial disc model and found that the source extension is $0.60 \pm 0.11$ with the $T_{\text {ext }}=224$, which corresponds to a significance of about $15 \sigma$. However, the log-likelihood is maximum for the Gaussian model; hence, it will be considered as the preferred model for 4FGL

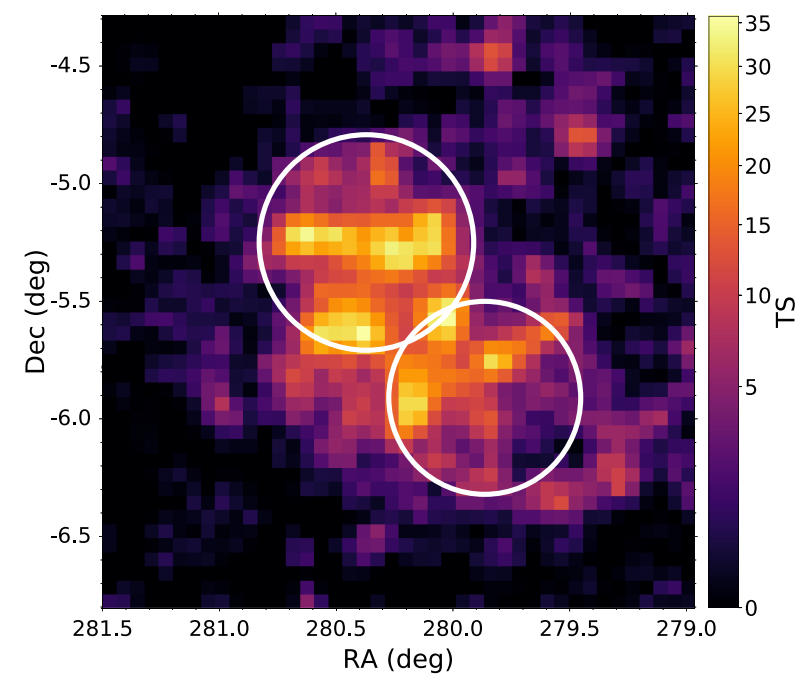

Figure 3. Fermi-LAT TS map (in Galactic coordinates) for the energy range from $10 \mathrm{GeV}$ to $1 \mathrm{TeV}$. The two FGES sources are also shown (white circles).

J1840.9-0532e. The resulting Fermi-LAT TS map above between $10 \mathrm{GeV}$ and $1 \mathrm{TeV}$ is shown on Fig. 3.

\subsubsection{Spectrum}

For the spectral study, we consider data within the energy range $10 \mathrm{GeV}-1 \mathrm{TeV}$. We calculate the SED of HESS J1841-055 using the best model obtained for the morphological study as discussed in Section 3.2.1. The SED of HESS J1841-055 is shown in Fig. 2, which is obtained by a fit to the data with the PL model.

The best-fitting PL model parameters are prefactor, $N_{0}=(1.71 \pm$ $0.41) \times 10^{-14} \mathrm{MeV}^{-1} \mathrm{~cm}^{-2} \mathrm{~s}^{-1}$, spectral index, $\alpha=2.30 \pm 0.03$, and scale, $E_{0}=1 \mathrm{GeV}$, where the uncertainties are statistical only. The total flux is found to be $F(>10 \mathrm{GeV})=(1.2 \pm 0.1) \times$ $10^{-8}$ photons $\mathrm{cm}^{-2} \mathrm{~s}^{-1}$.

\subsection{Joint fit to MAGIC and Fermi-LAT data}

We perform a joint likelihood fit to the observed fluxes from MAGIC and Fermi-LAT to find out the spectral behaviour of the source in the $\mathrm{GeV}-\mathrm{TeV}$ energy range. We perform a $\chi^{2}$ fit on the Fermi-LATMAGIC spectral points. We consider different spectral shapes as a PL, a PL with exponential cutoff (PLE), and a broken powerlaw (BPL) as spectral shapes for the fit. The PL has already been defined in subsection 3.1.2. The PLE spectral shape is defined 
Table 4. Best-fitting model parameters for the joint fit to MAGIC and Fermi-LAT spectral data points for three different models.

\begin{tabular}{|c|c|c|c|c|c|c|c|}
\hline Model & $\begin{array}{l}\text { Amplitude } \\
\left(\times 10^{-7}\right)\end{array}$ & index 1 & index2 & $\begin{array}{l}e_{\text {cutoff }} \\
(\mathrm{TeV})\end{array}$ & $\begin{array}{l}e_{\text {break }} \\
(\mathrm{TeV})\end{array}$ & $\chi^{2} /$ d.o.f. & $p$-value \\
\hline PL & $2.65 \pm 0.21$ & $2.23 \pm 0.02$ & - & - & - & $196.4 / 10$ & $9.1 \times 10^{-37}$ \\
\hline BPL & $1.53 \pm 0.22$ & $1.91 \pm 0.05$ & $2.75 \pm 0.10$ & - & $0.037 \pm 0.005$ & $20.8 / 8$ & $7.8 \times 10^{-3}$ \\
\hline
\end{tabular}

Note. The maximum-likelihood method is used to perform the joint fit.

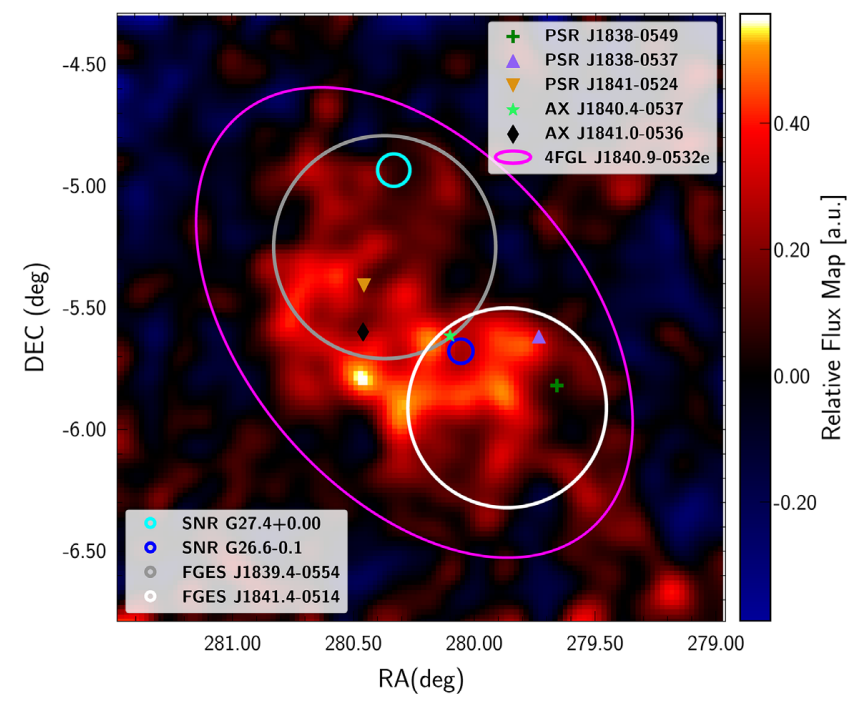

Figure 4. HE ( $>1 \mathrm{TeV}$ ) TS map as seen by MAGIC. Two extended FermiLAT sources FGES J1839.4-0554 and FGES J1841.4-0514 are overplotted (white and grey circles). The extension of the source reported in 4FGL catalogue is shown as a magenta ellipse. Other point-like sources present in this region are also displayed (different markers).

as

$$
\text { PLE : } \frac{\mathrm{d} N}{\mathrm{~d} E}=N_{0}\left(\frac{E}{E_{0}}\right)^{-\alpha} \exp \left(-\frac{E}{E_{0}}\right) \text {, }
$$

The BPL model is defined as follows:

$$
\text { BPL }: \frac{\mathrm{d} N}{\mathrm{~d} E}=\begin{array}{ll}
A\left(E / E_{0}\right)^{-\alpha_{1}} & : E<E_{\text {break }} \\
A\left(E_{\text {break }} / E_{0}\right)^{\alpha_{2}-\alpha_{1}}\left(E / E_{0}\right)^{-\alpha_{2}} & : E>E_{\text {break }}
\end{array},
$$

where $A, \alpha_{1}, \alpha_{2}$, and $E_{\text {break }}$ are parameters of the model. In the case of the BPL model, the spectral break is at $37 \mathrm{GeV}$, while the cutoff energy in the PLE model is located at $1.8 \mathrm{TeV}$. Both the BPL and PLE models describe the SED better than a simple PL model, implying that a significant curvature is present in the SED. However, both BPL and PLE models show similar fit probability ( $p$-value), making it difficult to favour one of them the most. A combined fit to the SED with both BPL and PLE is shown in Fig. 2. The parameters of the different models, tested with $\chi^{2} /$ d.o.f., are given in Table 4 .

\section{POTENTIAL COUNTERPARTS}

Several point-like sources lie in the FoV of the extended gammaray source HESS J1841-055 and are likely to contribute to the VHE emission. In this section, we discuss all these sources and their association with the observed emission. We consider some of the brightest emissions from these sources (see Fig. 4) discussed below to constrain the gamma-ray emission mechanisms in Section 5.

\subsection{G26.6-0.1}

The diffuse hard X-ray source G26.6-0.1 was detected by ASCA in a Galactic plane survey (Bamba et al. 2003), which is located in this region as shown in Fig. 4. The observed X-ray spectrum was found to be featureless and can be fitted with a PL function with photon index 1.3. The diffuse X-ray flux was estimated to be $3.5 \times 10^{-12} \mathrm{erg} \mathrm{cm}^{-2} \mathrm{~s}^{-1}$ from a radius of 12-arcmin region in the energy range of $0.7-7.0 \mathrm{keV}$. We consider this diffuse emission to be associated with HESS J1841-055 and assumed a corresponding scaled X-ray flux from a region with radius 0.4 similar to the extension of our source, for the multiwavelength (MWL) modelling in Section 5. The distance to G26.6-0.1 is $1.3 \mathrm{kpc}$ (Bamba et al. 2003). Following this, the distance of HESS J1841-055 is assumed to be $2 \mathrm{kpc}$.

\subsection{PSR J1838-0537, PSR J1841-0524, and PSR J1838-0549}

Several gamma-ray pulsars lie within the HESS J1841-055 region. Pletsch et al. (2012) discovered the gamma-ray pulsar PSR J1838-0537 in a blind search of Fermi-LAT data. It has been proposed as a potential candidate for the VHE source. It is a radioquiet pulsar. Also, no X-ray pulsation is observed from the location of the pulsar. If it is associated with a nebula, the subsequent observation from this region should have provided a detectable level of radio and $\mathrm{X}$-ray fluxes from this region. The spin-down power of the pulsar is estimated to be $\dot{E}=5.9 \times 10^{36} \mathrm{erg} \mathrm{s}^{-1}$. The integral energy flux of HESS J1841 -055 estimated by MAGIC over the range $0.1-10 \mathrm{TeV}$ is $l \gamma \sim 9.13 \times 10^{-11} \mathrm{erg} \mathrm{cm}^{-2} \mathrm{~s}^{-1}$. The luminosity for a distance of $2 \mathrm{kpc}, L_{\gamma}=4 \pi d^{2} l \gamma=4.37 \times 10^{34} \mathrm{erg} \mathrm{s}^{-1}$ for isotropic emission. This implies a conversion efficiency $\eta=L_{\gamma} / \dot{E} \sim 0.7$ per cent, which is consistent with other suggested pulsar/pulsar wind nebulae (PWNe) associations (Hessels et al. 2008). Hence, the pulsar's energetic is likely to power a PWN producing part of the $\mathrm{TeV}$ emission. The spectral index derived $\sim 2.4$ is relatively soft in comparison to other PWNe detected at GeV energies by Fermi-LAT. Hence, part of the LE emission could have a different origin.

There are two other known pulsars PSR J1841-0524 and PSR J1838-0549 (Aharonian et al. 2008), which can contribute to the emission of HESS J1841-055. The estimated $\dot{E} / D^{2}$ values are given as $4.4 \times 10^{33}$ and $4.7 \times 10^{33} \mathrm{erg} \mathrm{s}^{-1} \mathrm{kpc}^{-2}$, respectively, and they can contribute to the observed emission when considered together. However, if taken separately, each would require approximately 200 percent efficiency to explain the VHE emission (Aharonian et al. 2008). There is no significant radio emission observed from the location of these pulsars. The observed X-ray emission from these sources is lower than that considered for the multiwaveband modelling. Hence, assuming these constrains, we will not consider them separately for the MWL modelling.

Hence, out of the three pulsars located in the region, it is most likely that only PSR J1838-0537 is contributing to the detected gamma-ray emission. 


\subsection{FGES J1839.4-0554 and FGES J1841.4-0514}

Recent Fermi-LAT catalogue for Galactic extended sources (FGESs) shows that there are two distinct extended sources in this region with energies above $10 \mathrm{GeV}$ (Ackermann et al. 2017). These sources, FGES J1839.4-0554 and FGES J1841.4-0514, are located at RA, Dec. $=280.31 \pm 0.04,-5.22 \pm 0.03$, and $279.90 \pm 0.03$, $-5.90 \pm 0.03$ with an extension of $0.25 \pm 0.02$ and $0.31 \pm 0.03$, respectively. The extensions of these two sources are shown in Fig. 4. It is evident from the figure that the observed $\mathrm{GeV}$ emission is overlapping with the extension found at $\mathrm{TeV}$ energies. Therefore, they can be considered as potential counterparts for the $\mathrm{TeV}$ emission. Although it appears that there are two different sources, the spectral characteristics at energies above $10 \mathrm{GeV}$ are similar, indicating that they may have common origin (Ackermann et al. 2017). In our analysis of Fermi-LAT data in this paper, we consider the entire region that includes both these sources to estimate the SED and we use it for the MWL modelling.

\subsection{G27.4+0.00 (Kes 73)}

One of the sources studied in radio is a shell-type remnant Kes 73 (G027.4 + 00.0), which is present to the north-east of the extended emission region (see Fig. 4). The small diameter 5-arcmin radio shell is characterized by a steep spectral index $(\alpha \sim-0.68$, defined by $S \propto v^{\alpha}$ ) between 0.5 and $5 \mathrm{GHz}$ and flux density of $3.5 \pm 0.5 \mathrm{Jy}$ at $1.4 \mathrm{GHz}$ (Caswell et al. 1982). Radio studies of the remnant also show an incomplete shell structure with no central engine of Kes 73 and with a radio upper limits of 0.45 and $0.60 \mathrm{mJy}$ at 6- and $20-\mathrm{cm}$ radio wavelengths, respectively (Kriss et al. 1985). This is considered to be unlikely counterparts due to the very small angular size of $5 \mathrm{arcmin}$ and its location on the edge of the extended emission.

\subsection{AX J1840.4-0537 and AX J1841.4-0536}

A weak point-like source, 1RXS J184049.1-054336, is located within G26.6-0.1 and it contributes to less than 10 percent of the diffuse flux. Hence, it is reasonable to exclude this weak X-ray flux from our analysis. The other X-ray point sources AX J1840.4-0537 and AX J1841.4-0536 are located outside the G26.6-0.1 region but well within the extended HESS J1841-055. However the fluxes for these two sources were estimated to be $1.4 \times 10^{-13}$ and $2.1 \times 10^{-11} \mathrm{erg} \mathrm{cm}^{-2} \mathrm{~s}^{-1}$, respectively, which are below the level of the scaled diffuse $\mathrm{X}$-ray flux from this extended gamma-ray region. Moreover, due to the point-like morphology of these sources with no associated nebula around them, they can not be considered as potential counterparts of HESS J1841-055. However, a fraction of the total emission could be associated with these sources.

Hence, although it is challenging to disentangle which sources are contributing to the observed $\mathrm{GeV}-\mathrm{TeV}$ emission, we consider that the SNR G26.6-0.1, the pulsar PSR J1838-0537, and the extended FGES J1839.4-9554 and FGES J1841.4-0514 sources are the most promising counterparts, due to their energetics, extension, and location within the region.

\section{MODELLING OF THE SPECTRAL ENERGY DENSITY}

As already discussed above, there are several sources present in this extended region. Some of them are already argued to be potential counterparts at lower energies from the aspects of energetics of the system, while others are excluded due to their very point-like signatures along with the energetics that can not contribute significantly to the overall emission, considering the extent of the emission. In order to investigate if the MWL data can be explained selfconsistently, we consider that the observed emission is associated with HESS J1841-055. Since the radio and X-ray fluxes from the entire region of the extended emission can not be more than that estimated from different observations, we consider those results as upper limits in these frequencies after multiplying with a scaling factor attributed to the extended region and the emission regions from where corresponding radio and X-ray measurements are performed. For the GeV-TeV modelling, we will consider the Fermi-LAT and MAGIC data sets from this study and the HESS data points from Aharonian et al. (2008). We use the numerical code developed by Saha \& Bhattacharjee (2015) for the modelling.

\subsection{Leptonic model}

In Section 3.3, we found that the SED has a spectral curvature and can be better explained with either a BPL model or a PLE model. Since the observed gamma-ray spectra carry imprints of the intrinsic particle distribution, a single population of electrons that follows a BPL type of distribution of electrons is assumed to calculate the inverse Compton (IC) and bremsstrahlung emission processes.

In general, the electron spectrum might be more complicated than assuming a single population of electrons. For example, for the Crab Nebula, two different population of electrons are considered, namely, radio electrons and wind electrons. Radio electrons are less energetic electrons which reside in the nebular volume throughout its age, and they are mostly responsible for the observed radio fluxes. On the other hand, wind electrons are freshly accelerated electrons and they account for the observed fluxes at X-ray and $\mathrm{GeV}-\mathrm{TeV}$ energies. However, for simplicity, we consider a single population of electrons that is responsible for the observed emission at $\mathrm{GeV}-\mathrm{TeV}$ energies.

We first consider that the observed gamma-ray radiation at $\mathrm{GeV}-$ $\mathrm{TeV}$ energies is resulting from emission from relativistic electrons through IC and non-thermal bremsstrahlung processes. For IC process, we consider that the HE photons are produced by the upscattering of photons from the cosmic microwave background (CMB) and from interstellar dust contribution (Mathis, Mezger \& Panagia 1983). For bremsstrahlung process, we consider ambient matter density of $100 \mathrm{~cm}^{-3}$ following the estimation discussed in Appendix A. Higher or lower values of the ambient matter densities simply scale the contribution of bremsstrahlung spectrum. Fig. 5 shows both IC and bremsstrahlung spectra for the BPL electron distribution for an ambient matter density of $100 \mathrm{~cm}^{-3}$. The figure shows that the bremsstrahlung spectrum can explain the observed $\mathrm{SED}$ at $\mathrm{GeV}-\mathrm{TeV}$ energies. On the other hand, the IC emission for the target photons of CMB and star lights cannot explain the observed SED for the same population of electrons. The parameters of the BPL electron distribution are shown in Table 5. It is to be noted that the electron distribution can be adjusted to explain the observed emission by the IC spectrum. However, the bremsstrahlung spectrum will overestimate the observed flux for the same population of electron due to high ambient matter density. Hence, bremsstrahlung becomes dominant emission process within the leptonic scenario.

In order to check the contribution of the synchrotron spectrum for the electron distribution, we calculate the synchrotron spectrum leaving magnetic field as a free parameter. We find that the synchrotron spectrum for a magnetic field of approximately $5 \mu \mathrm{G}$ does not overestimate the radio and X-ray limits estimated for this study. The synchrotron component only contributes to radio and 


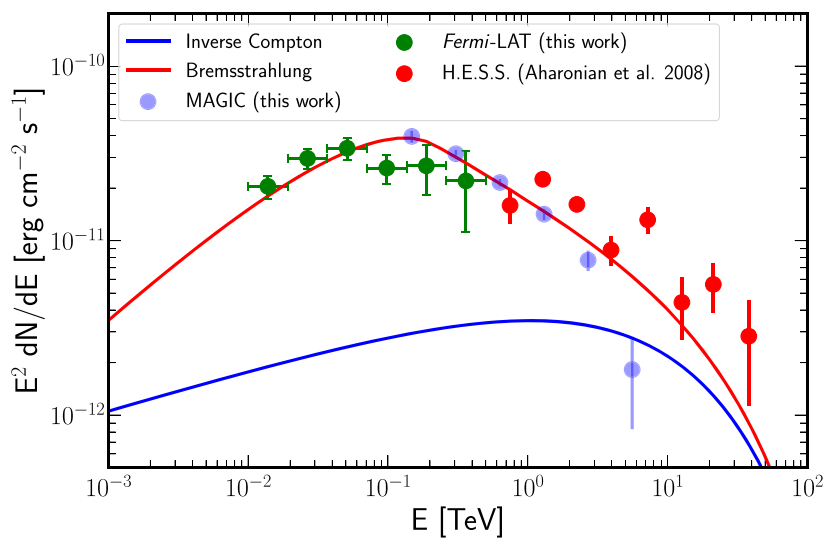

Figure 5. The SED of HESS J1841-055 fitted with a leptonic model where IC (blue line) and bremsstrahlung (red line) emissions are considered to account for fluxes at GeV-TeV energies, Fermi-LAT in green (this work), MAGIC in blue (this work), and HESS in red (Aharonian et al. 2008). Bremsstrahlung emission spectrum, estimated for an ambient matter density of $100 \mathrm{~cm}^{-3}$, is the dominant one. The parameters of the BPL electron distribution are shown in Table 5.

Table 5. Parameters for physical models for a single zone particle distribution of a BPL model.

\begin{tabular}{lcc}
\hline Parameters & Leptonic & Hadronic \\
\hline Spectral index $\left(\alpha_{1}\right)$ & $1.06_{-0.05}^{+0.10}$ & $1.18_{-0.11}^{+0.13}$ \\
Spectral index $\left(\alpha_{2}\right)$ & $2.52_{-0.05}^{+0.06}$ & $2.02_{-0.05}^{+0.05}$ \\
Energy at spectral break, $E_{\mathrm{b}}(\mathrm{TeV})$ & $0.18_{-0.02}^{+0.03}$ & $0.22_{-0.04}^{+0.07}$ \\
Ambient matter density, $n_{0}\left(\mathrm{~cm}^{-3}\right)$ & 100 & 100 \\
Total energy $\left(10^{48} \mathrm{erg}\right)$ & $5.82_{-0.19}^{+0.11}$ & $5.52_{-0.13}^{+0.12}$ \\
\hline
\end{tabular}

Notes. The parameters are obtained considering two different models: leptonic and hadronic. Parameters with errors are used as free parameters for the fit.
X-ray energies. The synchrotron spectrum together with IC and bremsstrahlung spectra is shown in Fig. 6.

\subsection{Hadronic model}

We also introduce a hadronic scenario as an additional component that contributes significantly at gamma rays $(\mathrm{GeV}-\mathrm{TeV})$. We calculate the gamma-ray spectrum resulting from the decay of neutral pions following Kelner, Aharonian \& Bugayov 2006. The gamma-ray spectrum for the relativistic protons for the BPL model as considered for the leptonic model and for an ambient gas density of $n_{0} \simeq$ $100 \mathrm{~cm}^{-3}$ is shown in Fig. 7. The total energy can be calculated as $W_{\mathrm{p}}=5.52 \times 10^{48} \times\left(100.0 / n_{0}\right) \mathrm{erg}$. The figure displays that the gamma-ray spectrum resulting from the decay of neutral pions can explain the observed $\mathrm{GeV}-\mathrm{TeV}$ data very well. The parameters of the model are presented in Table 5 .

\section{DISCUSSION}

\subsection{Gamma rays from extended unidentified sources}

The analysis of about $34 \mathrm{~h}$ of good-quality MAGIC data confirms that the gamma-ray emission is as extended as claimed by the HESS Collaboration and the source is detected with high significance for energy above $50 \mathrm{GeV}$. In addition to that, we investigate the source morphology as a function of energy. The observed results suggest that at low energies, the overall region is detected like a diffuse source, with some few regions around the centre of the source where the significance is higher than $5 \sigma$. This indicates the possibility that several point-like sources are contributing to the extended emission. At medium energies, between $500 \mathrm{GeV}$ and $1 \mathrm{TeV}$, the emission is concentrated along the centre, in a northsouth line. In addition to that, the skymap above $1 \mathrm{TeV}$ shows that the extension of the emission is reduced compared to that of low energies and the most significant flux is located at the southern part of the extended region, with only few hotspots over $5 \sigma$. The morphological analysis of MAGIC data also shows that the multiple

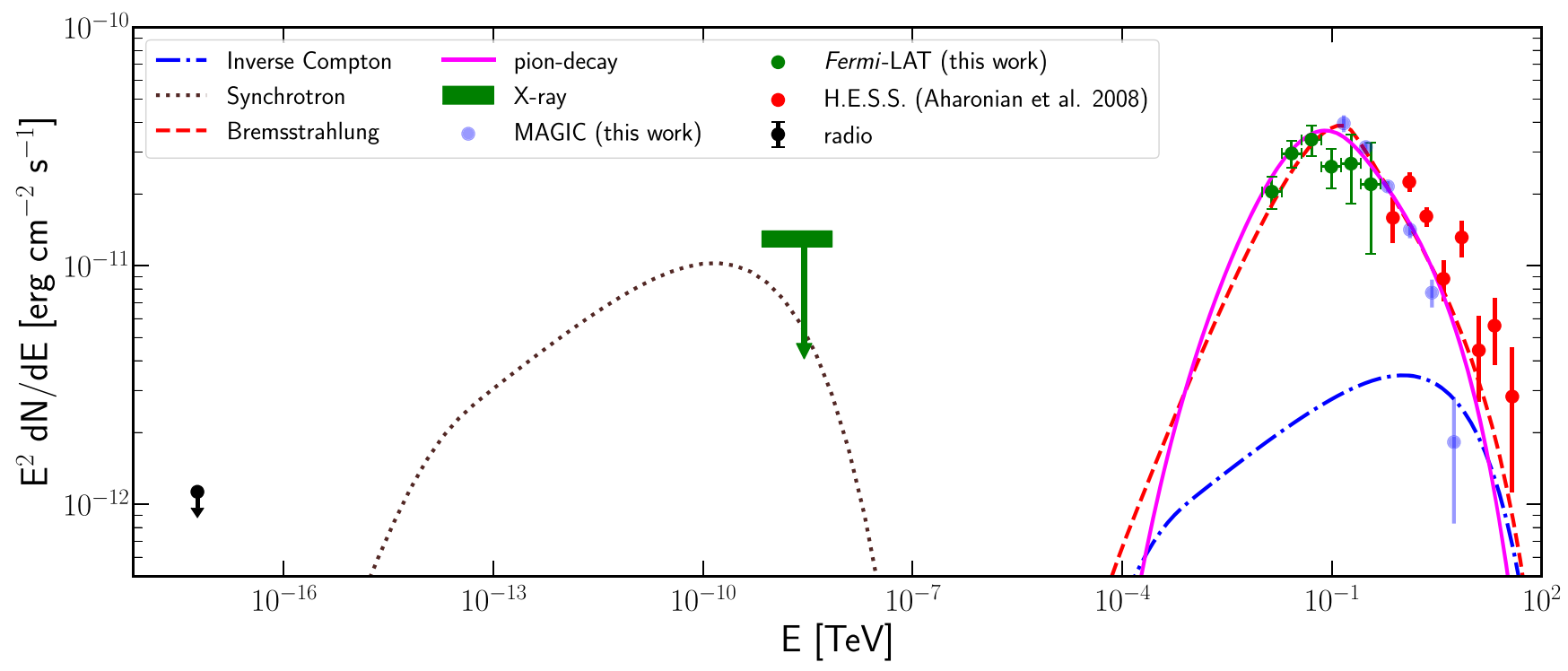

Figure 6. SED of HESS J1841-055 from radio to TeV energies. HE Fermi-LAT data (green points) and TeV MAGIC (blue points) and HESS (red points) data are fitted both with hadronic (magenta solid line) and bremsstrahlung (red dashed line) models. IC (blue dot-dashed line) cannot account for the measured gamma-ray emission. The synchrotron spectrum (brown dotted line) is fitted according to the radio and X-ray ULs. 


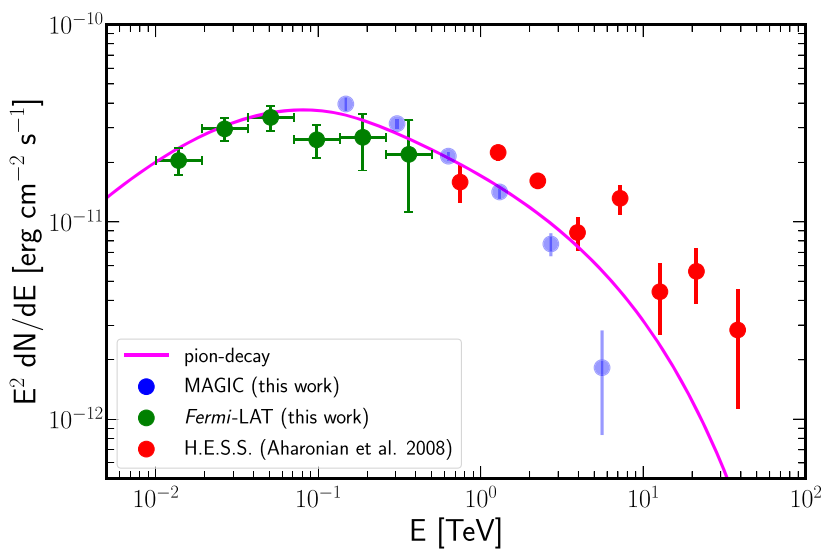

Figure 7. SED of HESS J1841-055, fitted with a $\pi^{0}$-decay emission spectrum, assuming the parameters shown in Table 5.

source model is better over a single-source model. This establishes the fact that several sources are contributing to the extended emission.

The morphological study of the source using about $10 \mathrm{yr}$ of FermiLAT data above $10 \mathrm{GeV}$ also shows that the source is extended. However, the spectral shape is different from that of MAGIC. When comparing with the emission at higher $(\mathrm{TeV})$ energies, HESS J1841-055 displays an extension compatible to that measured by MAGIC.

In the case of the Fermi-LAT detection, the spectrum of the source is best described by a PL. To study the spectral behaviour within the entire energy range, from $\mathrm{GeV}$ to $\mathrm{TeV}$ energies, we performed a joint fit on the spectral data points from MAGIC and Fermi-LAT. We found that the combined SED is best described either by a BPL model with a spectral break at $\sim 37 \mathrm{GeV}$ or with a PL with exponential cut-off at $1.8 \mathrm{TeV}$

\subsection{Emission mechanisms}

MWL modelling of the data indicates that the leptonic model can explain the data well. Due to the higher ambient matter density, the bremsstrahlung spectrum dominates over IC spectrum. The radio and X-ray fluxes put a constraint on the magnetic field in the emission volume when they are accounted with a synchrotron emission process. The magnetic field of $5 \mu \mathrm{G}$, as mentioned in Section 5.1, is very close to that of some other known old PWNe (Reynolds, Gaensler \& Bocchino 2012; Kargaltsev, Rangelov \& Pavlov 2013). Given the high ambient matter density and presence of molecular clouds, a hadronic emission model is also suitable to explain the observed data at $\mathrm{GeV}-\mathrm{TeV}$ energies. We find that the hadronic model can explain the data very well for a BPL proton distribution and an ambient matter density of $100 \mathrm{~cm}^{-3}$. Therefore, both leptonic and hadronic model can explain the data well with the parameters shown in Table 5.

In the whole discussion on MWL modelling of the data, our assumption was that the observed emission is entirely due to a single source. However, we have already seen that the region is populated by different sources that were established through observations at lower energy bands. Some of the sources are already excluded to be considered as potential gamma-ray emitters while energetic is considered. However, some of them could be potentially associated with the observed emission at $\mathrm{GeV}-\mathrm{TeV}$ energies. Given the angular resolution of the gamma-ray telescopes at present generation, it is not possible to have an unambiguous association with the sources at other wavebands. One possible scenario for the extension of the emission is the interaction of run-away cosmic particles from the source and the gamma-ray visibility is enhanced due to interaction with molecular clouds that are covering the extended source very well as can be seen from Fig. 8 and discussed in Appendix A. The presence of molecular clouds along the extension of the source also supports the relatively high ambient matter density required for both the leptonic and hadronic model.

\subsection{The nature of HESS J1841-055}

The observations at X-ray energies did not show any bright synchrotron nebula around the pulsars present in this region. However, in this scenario, the absence of bright synchrotron nebula can be easily explained. If the $\mathrm{TeV}$ source is powered by one or several pulsars present in this region, then pulsars are expected to be relic ones. For such PWNe, IC emission efficiency is more pronounced due to lower magnetic fields. In Section 5, we find that the IC contribution for this source is 10 per cent less compared to the bremsstrahlung spectrum. Therefore, it is reasonable to consider that the synchrotron emission could be even more inefficient, which supports the absence of the bright synchrotron nebula around the pulsars. Therefore, if the bright $\mathrm{TeV}$ emission is assumed to be associated with a PWN, then the PWN requires to be a relic one where the remnant of the supernova explosion has already disappeared.

It is also discussed in Section 4 that, energetically, the pulsar PSR J1838-0537 is able to account for the observed GeV-TeV energies. The gamma-ray flux at $\mathrm{TeV}$ energies, a factor of 2 lower than the Crab nebula flux, is required to have $S_{0}=\left(L_{0} / 10^{37} \mathrm{erg} \mathrm{s}^{-1}\right)(d / 1 \mathrm{kpc})^{-2} \geq$ $10^{-3}$, where $L_{0}$ and $d$ are the luminosity and distance of the source, respectively (Aharonian 2004). The two known pulsars PSR J1841-0524 and PSR J1838-0549 cannot contribute to the observed $\mathrm{GeV}-\mathrm{TeV}$ energies since $S_{0}$ is less than $10^{-3}$. However, $S_{0}$ is greater than $10^{-3}$ for PSR J1838-0537, making it a potential counterpart of HESS J1841-055. Since PSR J1838-0537 is not a part of any radio or X-ray nebula, it is also possible to consider that it is an isolated pulsar that has already left the remnant. $\mathrm{TeV}$ emission is an effective product of the IC mechanism for such isolated pulsars, with the injection of relativistic electrons in the interstellar magnetic field, which is about $3 \mu \mathrm{G}$. In such a scenario, the bright X-ray and radio synchrotron nebula could be absent. However, the extension of such a source is not readily accepted. Nevertheless, the presence of the molecular clouds along the observed $\mathrm{GeV}-\mathrm{TeV}$ emission can support its extension within this scenario but through bremsstrahlung processes.

The extension of the source is estimated to be 0.4 when the source is fitted with a 2D Gaussian with a equal spatial width for both the directions. This extension translates into a radius of approximately $14 \mathrm{pc}$ at a source distance of $2 \mathrm{kpc}$. The effective diffusion radius can be calculate as, $R_{\text {diff }} \simeq 2 \sqrt{D(E) t}$, where $D(E)$ is the diffusion coefficient and can be represented as $D(E)=D_{0}(E / 10 \mathrm{GeV})^{\delta}$ (Atoyan, Aharonian \& Völk 1995). The commonly used diffusion coefficient at $10 \mathrm{GeV}$ is of about $D_{0} \sim 10^{28} \mathrm{~cm}^{2} \mathrm{~s}^{-1}$ (Berezinsky et al. 1990) and assuming that $\delta=0.5$ ( $\delta$ is one of the parameters of the diffusion coefficients; for energy-independent diffusion coefficient, $\delta=0$ ), we calculate the diffusion time-scale of $t_{\text {diff }}=17 \mathrm{kyr}$. On the other hand, the lifetime of the bremsstrahlung loss, which is independent of energy, is estimated as $t_{\text {brems }} \simeq 4 \times 10^{4}\left(n / 1 \mathrm{~cm}^{-3}\right)^{-1} \mathrm{kyr}=4 \times 10^{2}$ kyr for ambient matter density of $100 \mathrm{~cm}^{-3}$. Therefore, the dominant emission through bremsstrahlung process for the estimated ambient matter density is a viable solution for the observed extension of 


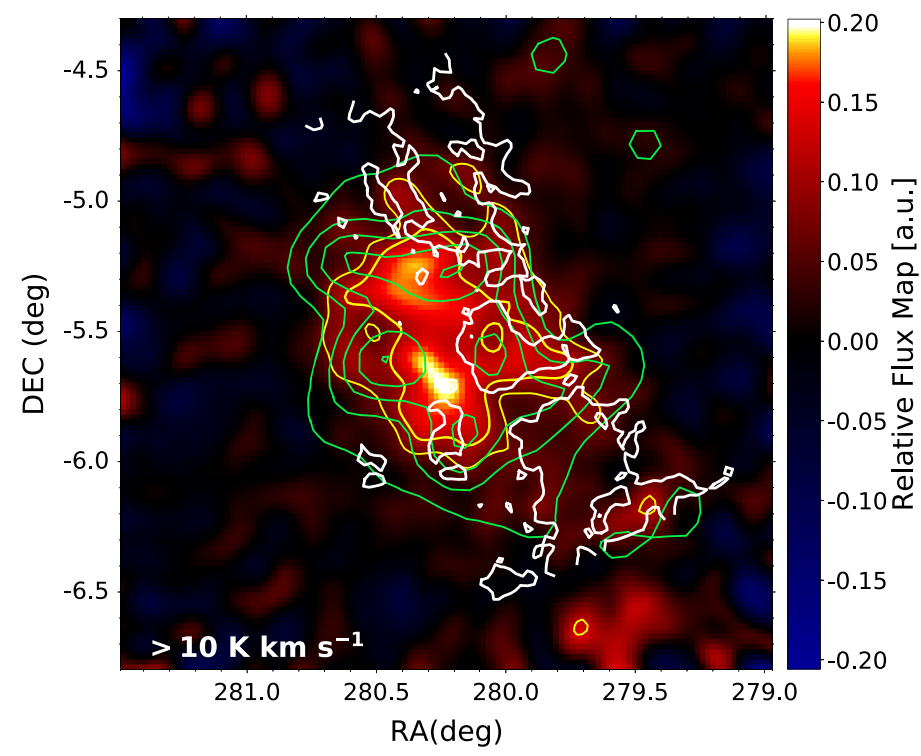

Figure 8. LE skymap, similar to Fig. 1. The source as seen by MAGIC is shown as yellow contours, while the Fermi-LAT source is plotted as green contours The CO contours are shown with white solid lines when CO map is integrated over the range of -5 to $135 \mathrm{~km} \mathrm{~s}^{-1}$. The CO data are obtained from archival Galactic Ring Survey (GRS; Jackson et al. 2006).

the source. We then conclude that the observed emission can be potentially associated with a PWN.

The observed emission can also be considered to be associated with SNRs, since it is seen that there are two SNRs present in and around the source. The first one, G27.4+0.00, is located at the edge of the $\mathrm{TeV}$ emission and has a relatively small angular size; hence, it is unlikely that it can account for the observed gamma rays. G26.6-0.1 is considered to be a potential counterpart for the extended emission. However, there are no strong radio and X-ray nebulae associated with the extent of the emission, which is the case for a typical SNR scenario. Hence, a strong association can be made, provided that the observed emission is considered due to the particles that escaped the SNR shocks and are interacting with the molecular clouds. Following the diffusion time-scale as discussed in the preceding paragraph and the age of the SNR G26.6-0.1 as a middle-age SNR ( $\sim 10^{3} \mathrm{yr}$; Bamba et al. 2003), it can be considered a possible candidate for at least part of the detected $\mathrm{GeV}-\mathrm{TeV}$ emission

\section{SUMMARY AND CONCLUSIONS}

We report a deep study of the unidentified gamma-ray source HESS J1841-055 at GeV-TeV energies using about $34 \mathrm{~h}$ of MAGIC and $10 \mathrm{yr}$ of Fermi-LAT data. We summarize the results as follows:

(i) The results of the detailed analysis show that the observed gamma-ray emission from HESS J1841-055 is significantly extended. The estimated extension of the source using MAGIC data is similar to that reported by the HESS Collaboration, found to be $\sim 0.4$, assuming a Gaussian distribution.

(ii) There are several bright hotspots in the extension of the source that appears to be multiple sources which contribute to the observed emission at $\mathrm{GeV}-\mathrm{TeV}$ energies. The emission at $\mathrm{TeV}$ energies moves towards the south with increasing energy, revealing this region as one of the potential main contributors of the $\mathrm{TeV}$ extended emission. (iii) The extended emission is modelled better with a multisource model compared to a single-source model.

(iv) The spectral curvature of the SED in the energy range from $\mathrm{GeV}-\mathrm{TeV}$ is significant and it can either be described by a BPL model with break at $37 \mathrm{GeV}$ or a PL with exponential cut-off at 1.8 $\mathrm{TeV}$.

(v) The observed SED can be explained well with both a leptonic (bremsstrahlung) and a hadronic model for the density of ambient matter of $100 \mathrm{~cm}^{-3}$, assuming a BPL distribution of electrons and protons, respectively.

Within the present morphological and spectral studies of this extended source using $\mathrm{GeV}-\mathrm{TeV}$ data and available MWL information on sources present within the region, we conclude that the extended gamma-ray emission seems to be associated with multiple sources in this region. The $\mathrm{GeV}-\mathrm{TeV}$ emission is compatible with a PWN scenario, although a fraction of the gamma-ray emission can also be explained within an SNR scenario. However, disentangling these sources at $\mathrm{TeV}$ energies (either point sources or extended sources) from one another and quantifying their contribution to the observed morphology of the source demands much better angular resolution compared to the present generation of gamma-ray telescopes. Hence, it becomes naturally an interesting source of study for the next generation of IACT telescopes.

\section{ACKNOWLEDGEMENTS}

We would like to thank the Instituto de Astrofisica de Canarias for the excellent working conditions at the Observatorio del Roque de los Muchachos in La Palma. The financial support by the German BMBF and MPG; the Italian INFN and INAF; the Swiss National Fund SNF; the ERDF under the Spanish MINECO (FPA201569818-P, FPA2012-36668, FPA2015-68378-P, FPA2015-69210-C62-R, FPA2015-69210-C6-4-R, FPA2015-69210-C6-6-R, AYA201571042-P, AYA2016-76012-C3-1-P, ESP2015-71662-C2-2-P, and FPA2017-90566-REDC); the Indian Department of Atomic Energy; 
and the Japanese JSPS and MEXT; the Bulgarian Ministry of Education and Science, National RI Roadmap Project DO1-153/28.08.2018 and the Academy of Finland grant nr. 320045 is gratefully acknowledged. This work was also supported by the Spanish Centro de Excelencia 'Severo Ochoa' SEV-2012-0234 and SEV-2015-0548, and Unidad de Excelencia 'Maria de Maeztu' MDM-2014-0369, by the Croatian Science Foundation (HrZZ) Project 09/176 and the University of Rijeka Project 13.12.1.3.02, by the DFG Collaborative Research Centers SFB823/C4 and SFB876/C3, and by the Polish National Research Centre grant UMO-2016/22/M/ST9/00382 and by the Brazilian MCTIC, CNPq and FAPERJ.

This publication makes use of molecular line data from the Boston University-FCRAO Galactic Ring Survey (GRS). The GRS is a joint project of Boston University and Five College Radio Astronomy Observatory, funded by the National Science Foundation under grants AST-9800334, AST-0098562, AST-0100793, AST0228993, and AST-0507657.

\section{DATA AVAILABILITY}

Raw data were generated at the MAGIC telescope large-scale facility. Derived data supporting the findings of this study are available from the corresponding authors upon request. Proprietary data reconstruction codes were also generated at the MAGIC telescope large-scale facility. The Fermi-LAT data are available in the public domain https://fermi.gsfc.nasa.gov/ssc/data/access/. Information supporting the findings of this study is available from the corresponding authors upon request.

\section{REFERENCES}

Abdollahi S. et al., 2020, ApJS, 247, 33

Abeysekara A. U.et al., 2016, ApJ, 817, 3

Abeysekara A. U. et al., 2017, ApJ, 843, 40

Ackermann M.et al., 2017, ApJ, 843, 139

Aharonian F., 2004, Very High Energy Cosmic Gamma Radiation: A Crucial Window on the Extreme Universe. World Scientific, Singapore

Aharonian F.et al., 2008, A\&A, 477, 353

Ajello M. et al., 2017, ApJS, 232, 18

Aleksić J. et al., 2012, Astropart. Phys., 35, 435

Atoyan A. M., Aharonian F. A., Völk H. J., 1995, Phys. Rev. D, 52, 3265

Atwood W. B. et al., 2009, ApJ, 697, 1071

Bamba A., Ueno M., Koyama K., Yamauchi S., 2003, ApJ, 589, 253

Bartoli B. et al., 2013, ApJ, 767, 99

Berezinsky V. S., Bulanov S. V., Dogiel V. A., Ptuskin V. S., 1990, in Ginzburg V. L., ed., Astrophysics of Cosmic Rays. NorthHolland, Amsterdam

Caswell J. L., Haynes R. F., Milne D. K., Wellington K. J., 1982, MNRAS, 200, 1143

Dame T. M., Hartmann D., Thaddeus P., 2001, ApJ, 547, 792

Dickey J. M., Lockman F. J., 1990, ARA\&A, 28, 215

Fomin V. P., Stepanian A. A., Lamb R. C., Lewis D. A., Punch M., Weekes T. C., 1994, Astropart. Phys., 2, 137

HESS Collaboration et al., 2018, A\&A, 612, A1

Hessels J. W. T. et al., 2008, ApJ, 682, L41

HI4PI Collaboration et al., 2016, A\&A, 594, A116

Jackson J. M. et al., 2006, ApJS, 163, 145

Kargaltsev O., Rangelov B., Pavlov G. G., 2013, preprint (arXiv:1305.2552)

Kelner S. R., Aharonian F. A., Bugayov V. V., 2006, Phys. Rev. D, 74, 034018

Kriss G. A., Becker R. H., Helfand D. J., Canizares C. R., 1985, ApJ, 288, 703

Mathis J. S., Mezger P. G., Panagia N., 1983, A\&A, 128, 212

Moralejo A. et al., for the MAGIC Collaboration, 2009, Proceedings, 31st International Cosmic Ray Conference (ICRC2009): Lodz, Poland, 2009, p. 2693
Nobukawa K. K., Nobukawa M., Tsuru T. G., Koyama K., 2015, Adv. Space Res., 55, 2493

Pletsch H. J. et al., 2012, ApJ, 755, L20

Reynolds S. P., Gaensler B. M., Bocchino F., 2012, Space Sci. Rev., 166, 231

Saha L., Bhattacharjee P., 2015, J. High Energy Astrophys., 5, 9

Sguera V., Romero G. E., Bazzano A., Masetti N., Bird A. J., Bassani L., 2009, ApJ, 697, 1194

Vovk I., Strzys M., Fruck C., 2018, A\&A, 619, A7

Zanin R. et al., for the MAGIC Collaboration, 2013, Proceedings, 33rd International Cosmic Ray Conference (ICRC2013): Rio de Janerio, Brazil, 2013, p. 2937

\section{APPENDIX A: TARGET GAS DENSITY}

Here we evaluate the target gas density required both for leptonic and hadronic models as discussed in Sections 5.1 and 5.2. To evaluate the target gas density, we estimate the densities of each gas phase (neutral hydrogen $\mathrm{HI}$ and molecular hydrogen $\mathrm{H}_{2}$ ) and then sum the estimated values to get the total contribution to the gas density. Under the assumption of the optically thin limit, the HI column density is given by (Dickey \& Lockman 1990), $N(\mathrm{HI}) \simeq 1.823 \times 10^{18} \int T_{\mathrm{b}}\left(\mathrm{HI} ; v_{\mathrm{r}}\right) \mathrm{dv}_{\mathrm{r}} \mathrm{cm}^{-2}$, where $T_{\mathrm{b}}\left(\mathrm{HI} ; v_{\mathrm{r}}\right)$ is the brightness temperature of the observed $21-\mathrm{cm}$ line at $v_{\mathrm{r}}$. In order not to overestimate the gas density within the source, we need to integrate over some range of $v_{\mathrm{r}}$. We consider $v_{\mathrm{r}}$ in the range of $110-135 \mathrm{~km} \mathrm{~s}^{-1}$ corresponding to the distance of about $2 \mathrm{kpc}$. The average $\mathrm{HI}$ density is estimated to be $N(\mathrm{HI})=8.65 \times 10^{20} \mathrm{~cm}^{-2}$ for a radius of 0.4 centred on the HESS J1841-055 using the data base of the HI4 $\pi$ survey (HI4PI Collaboration et al. 2016). We assume that the $\mathrm{HI}$ gas is uniformly distributed within the source. The length of the line of sight along the direction of the HESS J1841-055 is $\ell=2 r_{0}$, where $r_{0}$ is the radius of the extended emission. The radius of the source is considered to be 0.4 , which translates into approximately $14 \mathrm{pc}$ for a distance of $2 \mathrm{kpc}$ to the source. The density of the neutral hydrogen gas is $n(\mathrm{HI})=N(\mathrm{HI}) / \ell \simeq 10 \mathrm{~cm}^{-3}$. We use observations (see Fig. 8) of the ${ }^{13} \mathrm{CO}(J=1-0)$ line, which traces molecular clouds, from the Galactic Ring Survey (GRS; Jackson et al. 2006). The $\mathrm{CO}$ spectrum over the range of velocities from +110 to $+135 \mathrm{~km} \mathrm{~s}^{-1}$ are integrated to obtain the velocity-integrated $\mathrm{CO}$ intensity $\left(W_{\mathrm{CO}}\right)$. The $W_{\mathrm{CO}}$ averaged over the region with a radius of 0.4 covering the extended emission is found to be approximately $63 \mathrm{~K} \mathrm{~km} \mathrm{~s}^{-1}$. To estimate the mass of the molecular cloud, we use the standard $\mathrm{CO}$ to $\mathrm{H}_{2}$ conversion factor of $X_{\mathrm{CO}}=N\left(\mathrm{H}_{2}\right) / W_{\mathrm{CO}}=$ $1.8 \times 10^{20} \mathrm{~cm}^{-2} \mathrm{~K}^{-1} \mathrm{~km}^{-1} \mathrm{~s}$ (Dame, Hartmann \& Thaddeus 2001). We find $N\left(\mathrm{H}_{2}\right)=4.8 \times 10^{22} \mathrm{~cm}^{-2}$. Therefore, the density of the molecular hydrogen gas, $n\left(\mathrm{H}_{2}\right)=N\left(\mathrm{H}_{2}\right) / \ell \simeq 130 \mathrm{~cm}^{-3}$. The total gas density, hence, is $n(\mathrm{HI})+n\left(\mathrm{H}_{2}\right) \simeq 140 \mathrm{~cm}^{-3}$. However, for simplicity, we consider the gas density of $100 \mathrm{~cm}^{-3}$ for the physical modelling of the source.

\footnotetext{
${ }^{1}$ Instituto de Astrofísica de Canarias, E-38200 La Laguna, Spain

${ }^{2}$ Università di Udine, and INFN Trieste, I-33100 Udine, Italy

${ }^{3}$ National Institute for Astrophysics (INAF), I-00136 Rome, Italy

${ }^{4}$ ETH Zurich, CH-8093 Zurich, Switzerland

${ }^{5}$ Japanese MAGIC Group: ICRR, The University of Tokyo, Kashiwa, 2778582 Chiba, Japan

${ }^{6}$ Technische Universität Dortmund, D-44221 Dortmund, Germany

${ }^{7}$ Croatian MAGIC Group: University of Zagreb, Faculty of Electrical Engineering and Computing (FER), 10000 Zagreb, Croatia

${ }^{8}$ Saha Institute of Nuclear Physics, HBNI, 1/AF Bidhannagar, Salt Lake, Sector-1, Kolkata 700064, India

${ }^{9}$ IPARCOS Institute and EMFTEL Department, Universidad Complutense de Madrid, E-28040 Madrid, Spain
} 
${ }^{10}$ Centro Brasileiro de Pesquisas Físicas (CBPF), 22290-180 URCA, Rio de Janeiro (RJ), Brazil

${ }^{11}$ Departamento Astrofísica, Universidad de La Laguna, E-38206 La Laguna, Tenerife, Spain

${ }^{12}$ Faculty of Physics and Applied Informatics, Department of Astrophysics, University of Lodz, PL-90-236 Lodz, Poland

${ }^{13}$ Università di Siena and INFN Pisa, I-53100 Siena, Italy

${ }^{14}$ Deutsches Elektronen-Synchrotron (DESY), D-15738 Zeuthen, Germany

${ }^{15}$ Centro de Investigaciones Energéticas, Meioambientales y Tecnológicas, E-28040 Madrid, Spain

${ }^{16}$ Istituto Nazionale Fisica Nucleare (INFN), I-00044 Frascati (Roma), Italy

${ }^{17}$ Max-Planck-Institut für Physik, D-80805 München, Germany

${ }^{18}$ Institut de Física d'Altes Energies (IFAE), The Barcelona Institute of Science and Technology (BIST), E-08193 Bellaterra (Barcelona), Spain

${ }^{19}$ Università di Padova and INFN, I-35131 Padova, Italy

${ }^{20}$ Università di Pisa, and INFN Pisa, I-56126 Pisa, Italy

${ }^{21}$ Universitat de Barcelona, ICCUB, IEEC-UB, E-08028 Barcelona, Spain

${ }^{22}$ Armenian MAGIC Group: ICRANet-Armenia at NAS RA, 0019, Yerevan, Armenia

${ }^{23}$ Croatian MAGIC Group: Department of Physics, University of Rijeka, 51000 Rijeka, Croatia
${ }^{24}$ Universität Würzburg, D-97074 Würzburg, Germany

${ }^{25}$ Finnish MAGIC Group: Finnish Centre of Astronomy with ESO, University of Turku, FI-20014 Turku, Finland

${ }^{26}$ Departament de Física, and CERES-IEEC, Universitat Autònoma de Barcelona, E-08193 Bellaterra, Spain

${ }^{27}$ Croatian MAGIC Group: University of Split, Faculty of Electrical Engineering, Mechanical Engineering and Naval Architecture (FESB), 21000 Split, Croatia

${ }^{28}$ Croatian MAGIC Group: University of Osijek, 31000 Osijek, Croatia

${ }^{29}$ Japanese MAGIC Group: Department of Physics, Kyoto University, 6068502 Kyoto, Japan

${ }^{30}$ Japanese MAGIC Group: Department of Physics, Tokai University, Hiratsuka, 259-1292 Kanagawa, Japan

${ }^{31}$ Institute for Nuclear Research and Nuclear Energy, Bulgarian Academy of Sciences, BG-1784 Sofia, Bulgaria

${ }^{32}$ Finnish MAGIC Group: Astronomy Research Unit, University of Oulu, FI90014 Oulu, Finland

${ }^{33}$ Croatian MAGIC Group: Rudjer Boskovic Institute, 10000 Zagreb, Croatia

This paper has been typeset from a $\mathrm{T}_{\mathrm{E}} \mathrm{X} / \mathrm{L} \mathrm{T} \mathrm{E} \mathrm{X}$ file prepared by the author. 